\title{
Michael Buchfelder and Federica Guaraldi (Eds): Imaging in Endocrine Disorders
}

\author{
S. Karger AG Basel, 2016, (ISBN 978-3-318-02737-2)
}

\author{
Mariarosaria Manganelli $^{1} \cdot$ Luigi Mansi $^{1}$
}

Published online: 25 January 2017

(C) Springer-Verlag Berlin Heidelberg 2017

Imaging of Endocrine Disorders is the 45th volume of the series Frontiers of Hormone Research, directed by Ezio Ghigo, from the University of Turin. This book is authored by many international experts, under the editorial responsibility of Michael Buchfelder, neurosurgeon at the University of Erlangen-Nurnberg, and Federica Guaraldi, endocrinologist at the University of Turin. The structure is based on concise and well-illustrated chapters on diagnostic imaging of all the organs involved in endocrine/metabolic disorders, discussed in 156 pages and enriched by many images.

The text is organized into 12 chapters, divided into several parts according to the discussed topic and the described methods. The first four chapters are dedicated to the thyroid and parathyroid. Chapter 1 discusses the role of ultrasonography, specifically in lesions that require differential diagnosis. The second chapter focuses on CT and MR: although they are not methods of choice in patients with thyroid and parathyroid diseases, they can add important information in studying complex cases, with a role more evident in the preoperative planning. Chapter 3 illustrates the role of nuclear medicine, more relevant at the diagnostic level in the characterization of benign lesions. The fourth chapter addresses the role of hybrid methods, i.e. PET/CT and SPECT/CT in differentiated thyroid carcinoma. The next three chapters concern the study of adrenals and the endocrine pancreas, starting in Chapter 5 with a discussion of endoscopic ultrasonography (EUS-FNA) in characterizing adrenal lesions and in the diagnosis and follow-up of pancreatic lesions in patients with MEN1; the role of CT and

Luigi Mansi

luigi.mansi@unina2.it

1 Università della Campania "Luigi Vanvitelli", Caserta, Italy
MR in identifying incidentaloma is discussed in Chapter 6, and the seventh chapter discusses the study of adrenal glands in nuclear medicine. In this contribution, either consolidated or more experimental radiopharmaceuticals are presented, including those used for PET/CT, such as ${ }^{18} \mathrm{~F}$ - FDG,${ }^{11} \mathrm{C}$-HED, ${ }^{18} \mathrm{~F}$ FDOPA, ${ }^{11} \mathrm{C}$ metomidate (useful to differentiate adrenocortical lesions from non-adrenocortical ones, and in diagnosis of Conn's adenoma), ${ }^{123}$ I-MIBG for diagnosis and ${ }^{131} \mathrm{I}$-MIBG for therapy of pheochromocytomas. Chapter 8 defines the role of US and MR, respectively, as first- and second-line methods in related endocrine disorders in gonadal disorders. The following chapters dwell on the importance of MR in differential diagnosis of sellar region lesions (Chapter 9), also considering the pros and cons in the use of intra-operative MR for the removal of pituitary adenomas (Chapter 10). Chapter 11 instead focuses on high-affinity radiotracers for dopamine D2 receptors and of radiolabeled somatostatin analogues in helping diagnosis and management of pituitary adenomas. The last chapter, the twelfth, deals with the topic of neuroendocrine tumors, describing the actually used imaging techniques and future prospects. In particular, it is well-explained how the major role of PET in these tumors is associated with ${ }^{68} \mathrm{Ga}$-DOTA peptide somatostatin analogues more than with ${ }^{18} \mathrm{~F}-\mathrm{FDG}$.

All the chapters are accompanied by many pictures, in color and in black and white, all extensively commented on, from tables and explanatory graphs of the text content. The clear language, the conversational tone, as well as the wealth of images that correlate to each covered topic, make this publication appealing not only to endocrinologists, radiologists, nuclear physicians and internists, but also to primary care physicians frequently involved in the management of patients with endocrine disorders. The easy didactic structure together with the wide content create interest also for residents and students of medicine. 